UT-12-05

\title{
Discriminating Minimal SUGRA and Minimal Gauge Mediation Models at the Early LHC
}

\author{
Shoji Asai ${ }^{1}$, Eita Nakamura ${ }^{1}$ and Satoshi Shirai ${ }^{2,3}$ \\ ${ }^{1}$ Department of Physics, University of Tokyo, \\ Tokyo 113-0033, Japan \\ ${ }^{2}$ Department of Physics, University of California, \\ Berkeley, CA 94720 \\ 3 Theoretical Physics Group, Lawrence Berkeley National Laboratory, \\ Berkeley, CA 94720
}

\begin{abstract}
Among various supersymmetric (SUSY) standard models, the gravity mediation model with a neutralino LSP and the gauge mediation model with a very light gravitino are attractive from the cosmological view point. These models have different scales of SUSY breaking and their underlying physics in high energy is quite different. However, if the sparticles' decay into the gravitino is prompt in the latter case, their collider signatures can be similar: multiple jets and missing transverse momentum. In this paper, we study the discrimination between these models in minimal cases at the LHC based on the method using the significance variables in several different modes and show the discrimination is possible at a very early stage after the discovery.
\end{abstract}




\section{Introduction}

Supersymmetric (SUSY) standard model (SM) is the most promising candidate for the physics beyond the standard model (BSM). Once its presence is inferred from experiments, the next problem is to search for the underlying mechanism of SUSY breaking. The scale of SUSY breaking is one of the most important clue for this search.

There are many proposed models with various SUSY breaking scales. A major candidate is the gravity mediation model with a medium SUSY breaking scale. In the model, the lightest SUSY particle (LSP) is the Bino-like neutralino. It is stable and becomes a good candidate of a WIMP dark matter (DM), if $R$-parity conservation assumed. At the LHC, the LSP is observed as a missing transverse momentum $\left(p_{T}\right)$. The missing $p_{T}$ serves as a distinctive signature as well as high- $p_{T}$ jets and other decay products coming from decay chains of produced SUSY particles.

Another attractive candidate is the gauge mediation (GM) model with a low SUSY breaking scale since it solves the SUSY flavor problem. The gravitino becomes the LSP in the GM model and the collider signatures strongly depend on the gravitino mass $m_{3 / 2}$, which is related to the SUSY breaking scale $\sqrt{F}$ as $m_{3 / 2}=F /\left(\sqrt{3} M_{P L}\right)$, and the nature of the next LSP (NLSP). In the case of the heavier gravitino, the SUSY event at the LHC involves a non-pointing NLSP decay [1, 2] or a long-lived NLSP [3], which becomes a characteristic signature. On the other hand, if the gravitino is very light, the NLSP decay is prompt and the gravitino is observed as a missing $p_{T}$. Then, the event signature resembles that of the gravity mediation model. From the cosmological point of view, such a very light gravitino with $m_{3 / 2} \lesssim 10 \mathrm{eV}$ is very attractive since the model is free from gravitino problems [4]. So the discrimination between the gravity mediation model and the GM model with a very light gravitino is an important problem. However, this discrimination is difficult. In principle, measuring the LSP mass provides good information for discrimination. But, to measure the LSP mass precisely, a large

integrated luminosity is required, and in some case, obtained result may have degenerated solutions.

In this paper, we concentrate on the minimal types of the gravity mediation model and the GM model. We call these models the mSUGRA model and mGMSB model, 
respectively. The NLSP in the mGMSB model becomes either the Bino-like neutralino

$\tilde{\chi}_{1}^{0}$ or the lighter slepton $\tilde{\ell}$ (stau $\tilde{\tau}_{1}$ ). In the former case photons from the Bino decays are produced and in the latter case leptons are produced, and the photons or leptons are expected to be the discriminant of the GM model. High energy photons are rarely produced in the gravity mediation model and there is no difficulty in discriminating the GM model with a Bino NLSP. However, leptons are also produced in the gravity mediation models and thus the discrimination has a more quantitative nature. Also, in the experiment, the signal events are smeared by the SM backgrounds and the effect of detector efficiencies. Furthermore, if the lightest neutralino mass rests between the lighter slepton and stau: $m_{\tilde{\tau}_{1}}<m_{\tilde{\chi}_{1}^{0}}<m_{\tilde{\ell}_{R}}$, the lepton emissions can be suppressed and/or hidden.

The purpose of this paper is to study this discrimination between the mSUGRA and the mGMSB models in the realistic experimental setup. We propose a simple method of discrimination and show the integrated-luminosity dependence of the degeneracy of models. The method is applicable at an early stage of the LHC soon after the discovery.

The organization of the paper is as follows: In the next section, we describe the parameter spaces and the mass spectrums of the models considered in this paper and introduce the collider signatures of these models. We present the result of our Monte Carlo simulation and show the idea of using significance variables of multiple modes in Section 3. In Section 4, we discuss the discrimination of the mGMSB and the mSUGRA models based on a test on significance variables. Section 5 is devoted for discussions and conclusions.

\section{2 mSUGRA vs mGMSB: event signatures}

In this section, we first review the parameter space of each model and discuss the LHC signatures of these models. Then we describe the idea of discriminating these models at an early stage at the LHC.

\subsection{Model parameters and mass spectrum}

In this paper, we compare the collider signatures of the mSUGRA model and the mGMSB model. First, we describe the model parameters of each model. 
The mSUGRA model represents a mass spectrum of the gravity-mediated SUSY breaking model assuming the flavor blindness for the scalar masses and the CP property. The model parameters are $m_{0}, m_{1 / 2}, A_{0}, \tan \beta$ and $\operatorname{sign} \mu$, which is the unified scalar mass, the unified gaugino mass, the universal $A$-term, the ratio of the Higgs VEVs and the sign of $\mu$, respectively. The low-energy mass spectrum is obtained by solving the renormalizationgroup equations with these input values at the GUT scale. The resulting LSP is typically the lightest neutralino or the lightest slepton. From the cosmological view point, we only consider the case of a neutralino LSP.

As a parameter space of the mGMSB model, we adopt a parametrization used in Ref. [5]. Here, the ratio between the gaugino mass and the sfermion mass is left free while the "GUT relations" among the gaugino masses and the sfermion masses are imposed. The $A$-term is set to be 0 and the $B$-term is tuned to give a correct low-energy electroweak parameters. The model parameters are $\Lambda_{s}, \Lambda_{g}, M_{\text {mes }}, \tan \beta$ and $\operatorname{sign} \mu$. The sfermion masses and the gaugino masses at the messenger mass scale $M_{\text {mes }}$ are given by

$$
\begin{aligned}
M_{a} & =\frac{\alpha_{a}}{4 \pi} \Lambda_{g} \\
m_{\phi_{i}}^{2} & =\Lambda_{s}^{2} \sum_{a}\left(\frac{\alpha_{a}}{4 \pi}\right)^{2} C_{a}(i),
\end{aligned}
$$

where $\alpha_{a}(a=1,2,3)$ are the SM gauge couplings and $C_{a}(i)$ are the quadratic Casimir coefficients for each field. The $\Lambda_{s}$ and $\Lambda_{g}$ are related to the parameters of the conventional mGMSB model parameters as

$$
\begin{aligned}
& \Lambda_{s}^{2}=2 N_{5} \Lambda^{2}, \\
& \Lambda_{g}=N_{5} \Lambda,
\end{aligned}
$$

where $N_{5}$ is the number of messengers in the $5+5^{*}$ representation and $\Lambda=F / M_{\text {mes }}$ is the ratio of the square of the SUSY-breaking scale $\sqrt{F}$ and $M_{\text {mes }}$, when $F / M_{\text {mes }}^{2} \ll 1$. So in other words, the parameter representation in Eqs. (11) and (2) is the same as the conventional one with an arbitrary (non-integer) number of messengers.

\section{2 mSUGRA signal}

The LSP is the lightest neutralino in the mSUGRA model as described in the previous section. Once the SUSY particle, mostly the gluino and the squarks, is produced it 
decays in a cascade down to the SM particles and the LSP. Since the stability of the LSP is guaranteed by the $R$-parity conservation, the produced LSPs then escape outside the detector and are observed as a missing $p_{\mathrm{T}}$.

Since the gluinos and the squarks are colored particles and the LSP is a color-neutral particle, the cascade decay products include jets, as well as other SM particles depending on the detailed form of the decay chain. There are many possible decay chains. For example, in the decay chain $\tilde{q}_{L} \rightarrow \tilde{\chi}_{0}^{2} q \rightarrow \tilde{\chi}_{0}^{1} \ell \ell q$, leptons are emitted as well as jets and missing $p_{\mathrm{T}}$. Likewise, the decay products may include leptons, tau-jets or $b$-jets as well as the generic signal of jets and missing momentum.

\section{3 mGMSB signal}

In the mGMSB model we are interested in, the LSP is the gravitino. As in the mSUGRA case, the produced SUSY particles decay into the gravitino LSP. In the mGMSB case, however, the role of the NLSP becomes more important.

The decay length of the NLSP into the gravitino LSP is given by

$$
c \tau \sim 20 \mu \mathrm{m}\left(\frac{m_{3 / 2}}{1 \mathrm{eV}}\right)^{2}\left(\frac{m_{\mathrm{NLSP}}}{100 \mathrm{GeV}}\right)^{-5}
$$

Since this is much longer than the one resulting from the SM interaction, a produced SUSY particle usually decays into the NLSP before it directly decays into the LSP. 1 Thus, the event signature of the mGMSB model strongly depends on which particle is the NLSP.

As mentioned in the previous section, the NLSP in the mGMSB model is either the lightest neutralino or the lightest slepton (stau). In the neutralino-NLSP case the NLSP emits a photon and in the slepton-NLSP case the NLSP emits a lepton with a sizable branching fraction. The typical mGMSB signal, therefore, contains multi-jets + missing + multi-photons or multi-jets + missing + multi-leptons.

The lightest slepton is the lighter stau $\tilde{\tau}_{1}$ and the NLSP in the slepton-NLSP case. So as well as the multi-leptons signal, we expect events with multi tau-jets. The multi-tau-jets

\footnotetext{
1 There are exceptional cases. One example is the case when the right-handed slepton mass is degenerated to the lighter stau mass for small $\tan \beta[6$. Another example is the case that the masses of the lightest neutralino and the lighter stau are degenerated for $\Lambda_{g} \sim \Lambda_{s}$.
} 
mode becomes more important in the large $\tan \beta$ case, since then the heavier sparticles' decays into staus becomes more dominant because of the kinematical preference and the enlarged coupling of the $\tilde{\tau}_{1}$ due to the large left-right mixing of the staus.

\subsection{Strategy for discrimination}

As described above, an important difference between the mSUGRA model and the mGMSB models arises from the important role of the NLSP decay in the mGMSB model. In the mGMSB model with a neutralino-NLSP, the signal event usually accompanies two photons, and in the case with a slepton-NLSP, the signal accompanies multi-leptons. Grounded on these facts, we compare the models by using the following modes: no lepton +2 jets, no lepton +4 jets, same-sign (SS) 2 leptons, 2 tau-jets and 2 photons modes. In table 1, we summarize the naively expected number of signal events in these modes

Table 1: Naively expected number of signal events. The mark $\odot$ indicates that a large number of signals are expected and the mode is qualitatively "good" for model search. Similarly, $\bigcirc, \triangle$ and $\times$ indicates that the corresponding mode is qualitatively "not bad", "not good" and "bad".

\begin{tabular}{|c|c|c|c|}
\hline mode & mSUGRA & mGMSB $\tilde{\tau}$-NLSP & mGMSB $\tilde{\chi}_{1}^{0}$-NLSP \\
\hline $0 \ell+(2$ jets $/ 4$ jets $)$ & $\odot$ & $\triangle$ & $\triangle$ \\
SS $2 \ell$ & $\bigcirc$ & $\odot$ & $\triangle$ \\
$2 \tau$ & $\triangle$ & $\odot$ & $\triangle$ \\
$2 \gamma$ & $\times$ & $\times$ & $\odot$ \\
\hline
\end{tabular}

for each model. For example, in the mSUGRA model, the no lepton $+(2$ jets $/ 4$ jets $)$ mode is expected to be the most viable and the modes of two leptons or tau-jets are the next, but the two photons mode is bad because photons are hardly expected in this model. Similarly, the two leptons mode or the two tau-jets mode is expected to be the most viable one for the mGMSB model with a slepton-NLSP and the two photons mode is the best mode for the mGMSB model with a neutralino-NLSP. Since the location of the viable mode differs for the three cases, we may distinguish these models by observing which of the four modes shows the most signal events or which does not.

However, we need sophistication to apply this idea in the realistic experimental set- 
up. First, we must include contamination by the SM backgrounds and the detection efficiencies. The signal events may be hidden unless we carefully reduce the SM background contribution, which might dilute the apparent differences of characteristic events. Further, there are a certain amount of misidentification and fake rate of particles by the detection systems. This effect is non-negligible in the case of many leptons and photons, and is also a source of dilution of the signal events. Second, we need a quantitative way to describe the distinctions among various models, which can incorporate the experimental errors.

In the next section, we perform a Monte Carlo simulation, which incorporates the SM backgrounds and the detection efficiencies. We use the significance variable (see Sec. 3.2 for the definition and explanation) to solve the above problems.

\section{3 mSUGRA vs mGMSB: simulation}

In this section, we first describe the set-up of our Monte Carlo simulation and introduce the significance variable. We also review the current experimental constraints. We then show the result of our simulation and discuss the possibility of discrimination with significances in several different modes.

\subsection{SM backgrounds and detection efficiencies}

For the multi-jets mode, the main SM backgrounds come from the QCD jets, $t \bar{t}$ and gauge boson production events. For the multi-lepton mode $t \bar{t}$ and $W+$ jets are the main background and for the multi-photon mode, the $\gamma \gamma$ events are. To estimate these backgrounds and other sub-leading backgrounds, we used the programs MC@NLO 3.42[7] (for $t \bar{t}, W W, W Z$ and $Z Z$ ), Alpgen 2.13[8] (for $W j, Z j$ and $W / Z+b \bar{b} / t \bar{t}$ ), MadGraph 4.1.44 [9] (for $t \bar{t} / W / Z+\gamma / \gamma \gamma$ ), and Pythia 6.4 [10] (for $\gamma \gamma$ and QCD jets). As for the detector simulation, we used the AcerDet 1.0 [11]. For the isolation criteria for leptons and photons, we used the default setting of AcerDet.

In our simulation, we are interested in the signals with leptons or photons. The detection efficiency and misidentification of these particles are important in estimating both the signal events and background events. In the following simulation, we include the 
misidentification and fake rates of the leptons, photons and jets. The rates we have used in the analysis are shown in Table 2, The values are taken from Ref. [20]. For the SM backgrounds, however, the efficiencies are chosen to give more conservative results than the actual experimental data.

Table 2: Misidentification and fake rates. The efficiencies are taken from Ref. [20]. For SM backgrounds, the efficiencies are chosen to give a conservative estimation.

\begin{tabular}{|c|c|c|c|c|c|c|c|c|c|}
\hline & $j \rightarrow e$ & $j \rightarrow \mu$ & $j \rightarrow \gamma$ & $\tau \rightarrow j$ & $j \rightarrow \tau$ & $e \rightarrow j$ & $\mu \rightarrow j$ & $e \rightarrow \gamma$ & $\gamma \rightarrow j$ \\
\hline SUSY & $0.3 \%$ & $0.3 \%$ & $0.02 \%$ & $60 \%$ & $1 \%$ & $27 \%$ & $30 \%$ & $3 \%$ & $20 \%$ \\
\hline SM BKG & $0.3 \%$ & $0.3 \%$ & $0.02 \%$ & $20 \%$ & $1 \%$ & $0 \%$ & $0 \%$ & $3 \%$ & $0 \%$ \\
\hline
\end{tabular}

\subsection{Significance}

Under a fixed set of event cuts, we estimate the event number of the signals $N_{s}$ and the backgrounds $N_{b}$. To compare many model points in several modes on the same footing, we use the significance variable $Z$, which is a function of $N_{s}$ and $N_{b}$. We use the significance incorporating both the statistical and systematic uncertainties of the backgrounds and adopt the one used in Ref. [20]. The systematic uncertainties of the backgrounds are taken to be $50 \%$ for the QCD multi-jet background and $20 \%$ for others.

Given the number of the signal events $N_{s}$ and the background events $N_{b}$ with the uncertainty $\delta N_{b}$, the significance is given by calculating the convolution of the Poisson distribution with some "posterior" distribution function. As the posterior distribution, we take the gamma distribution as suggested in Ref. [12]. The resulting significance $Z$ is given by 12

$$
Z=\sqrt{2} \operatorname{erf}^{-1}(1-2 p)
$$

with

$$
p=\frac{B\left(N_{s}+N_{b}, 1+N_{b}^{2} / \delta N_{b}^{2}, \delta N_{b}^{2} /\left(N_{b}+\delta N_{b}^{2}\right)\right)}{B\left(N_{s}+N_{b}, 1+N_{b}^{2} / \delta N_{b}^{2}\right)}
$$

where $\operatorname{erf}^{-1}$ is the inverse error function and

$$
B(a, b, x)=\int_{0}^{x} d t t^{a-1}(1-t)^{b-1}
$$


is the incomplete beta function and $B(a, b)=B(a, b, 1)$ is the usual beta function. If we take the limit $\delta N_{b} \rightarrow 0$, the Eq. (7) reduces to the probability in the Poisson distribution. The statistical error of the significance $\delta Z_{B}$ is depends on both $N_{s}$ and $N_{b}$. The error is $\mathcal{O}(1)$ for the moderate values which we are interested in.

\subsection{Experimental constraints}

Up to now, the most stringent experimental constraints are given by the ATLAS and CMS experiments at integrated luminosities about $1 \mathrm{fb}^{-1}$. Analyses relevant for the GMSB and mSUGRA models are searches of jets with no lepton [13] or one lepton [14] by the ATLAS collaboration and searches of same-sign [15] or opposite-sign [16] dilepton and photons [17] by the CMS collaboration.

We show the constraints given by these experimental data in Fig. 1. To obtain the constraints, we apply the $95 \%$ C.L. exclusion limits given by the above analyses to our model points. To incorporate the NLO corrections to the cross section, we multiply a uniform $k$-factor of 1.5 for all model points, except for the mGMSB model points with a Bino-NLSP, at which the dominant contribution to the SUSY production is given by the direct Wino production.

\subsection{Signal events}

For both the mSUGRA and mGMSB models, the MSSM mass spectrums and the decay tables are calculated by using the program ISAJET 7.72 [18]. The SUSY events are generated by using the Herwig 6.510 [19] and we have used the AcerDet 1.0 [11] for the detector simulation.

For the mSUGRA model, the grid in parameter space is taken to be $0 \leq m_{0} \leq 1200$ $\mathrm{GeV}$ (in steps of $20 \mathrm{GeV}$ ), $100 \leq m_{1 / 2} \leq 600 \mathrm{GeV}$ (in steps of $20 \mathrm{GeV}$ ), $A_{0}=0, m_{0},-m_{0}$, $\tan \beta=10,40$ and $\operatorname{sign} \mu=+$. For the mGMSB model, the grid in parameter space is $-(100)^{2} \leq \Lambda_{s}^{2} \leq(400)^{2} \mathrm{TeV}^{2}$ (in steps of $5 \mathrm{TeV}$ ), $(100)^{2} \leq \Lambda_{g}^{2} \leq(400)^{2} \mathrm{TeV}^{2}$ (in steps of $5 \mathrm{TeV})$ and $\tan \beta=10,40$. The messenger mass scale is fixed as $m_{\text {mes }}=200 \mathrm{TeV} 2$ and the gravitino mass is set $m_{3 / 2}=16 \mathrm{eV}$.

\footnotetext{
${ }^{2}$ To avoid a messenger tachyon, the condition $F \lesssim m_{\text {mess }}^{2}$ is required, which indicates $m_{\text {mess }} \sim 100$ $\mathrm{TeV}$.
} 


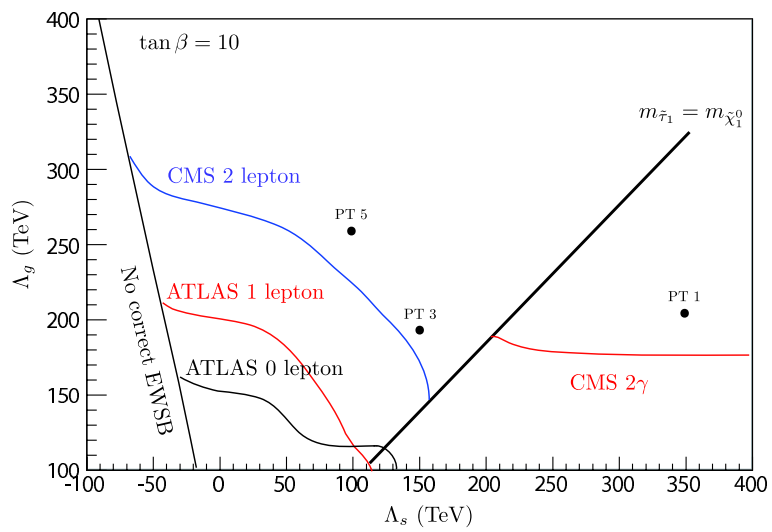

(a) mGMSB $\tan \beta=10$.

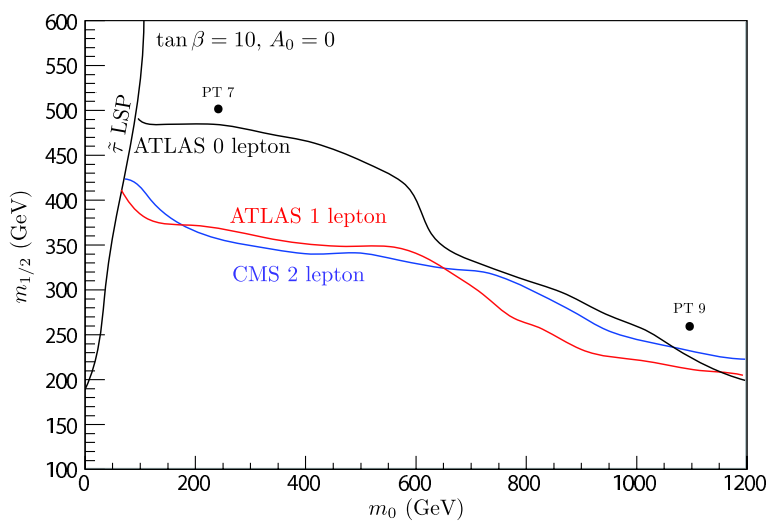

(c) mSUGRA $\tan \beta=10, A_{0}=0$.

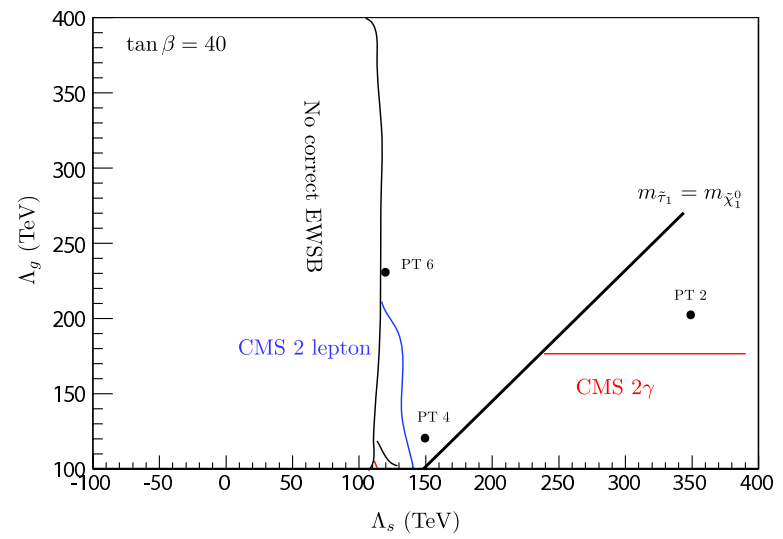

(b) mGMSB $\tan \beta=40$.

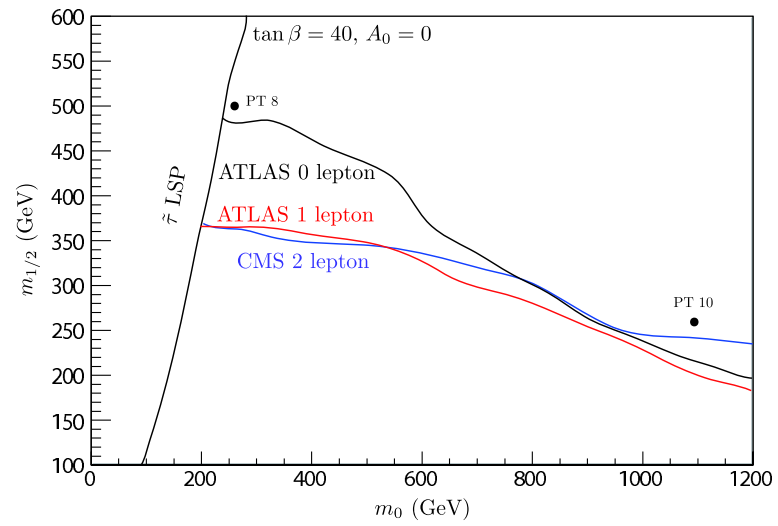

(d) mSUGRA $\tan \beta=40, A_{0}=0$.

Figure 1: Experimental constraints given by the LHC experiment at an integrated luminosity of about $1 \mathrm{fb}^{-1}$ at the $7 \mathrm{TeV}$ run. We also show the benchmark points PT1-10. 
Because we are interested in the model points that are potentially discovered at an early stage of the LHC and are not excluded by the experiments, we select model points within a certain mass region to reduce the size of computational calculation. For the mSUGRA, we select model points with $800<m_{\tilde{d}_{R}}<1600 \mathrm{GeV}$ and $400<m_{\tilde{g}}<1400$ $\mathrm{GeV}$. For the mGMSB, we select model points with $850<m_{\tilde{d}_{R}}<1600 \mathrm{GeV}$ for the slepton-NLSP case and $220<m_{\tilde{\chi}_{1}^{0}}<350 \mathrm{GeV}$ for the neutralino-NLSP case. The number of model points after this selection is 5633 (4046) for the mSUGRA (mGMSB) model. After imposing the experimental constraints reviewed in the previous section the number reduces to 3654 (3048).

\subsection{Modes and kinematical cuts}

In the following analysis, we use five different modes, no lepton modes with two and four jets, two photons mode, two $\tau$-jets mode, and the same-sign (SS) two leptons mode.

The kinematical cuts for these search modes are summarized in Table 3. In the table,

Table 3: List of kinematical cuts used in the analysis. The $E_{\mathrm{T}}^{\mathrm{miss}}$ is the absolute value of the missing $p_{\mathrm{T}}$ and $M_{\mathrm{eff}}$ is defined in Eq. (9). We require isolation of leptons and photons (see text). In the four jets with no lepton mode, the values in brackets are chosen for each model point to optimize the significance.

\begin{tabular}{|c|c|}
\hline mode & \\
\hline $\mathrm{SS} 2 \ell$ & $\begin{array}{l}\text { At least two leptons with } p_{\mathrm{T}}>20 \mathrm{GeV} \text {. The leading two leptons have } \\
\text { the same charge. At least } 2 \text { jets with } p_{\mathrm{T}}>50 \mathrm{GeV} \text { and for the leading } \\
\text { jet } p_{\mathrm{T}}>100 \mathrm{GeV} . E_{\mathrm{T}}^{\text {miss }}>100 \mathrm{GeV}, M_{\mathrm{eff}}>800 \mathrm{GeV} \text {. }\end{array}$ \\
\hline $2 \tau$ & $\begin{array}{l}\text { At least two tau-jets with } p_{\mathrm{T}}>40 \mathrm{GeV} . \text { At least } 4 \text { jets with } \\
p_{\mathrm{T}}>50 \mathrm{GeV} \text { and for the leading jet } p_{\mathrm{T}}>100 \mathrm{GeV} . \\
E_{\mathrm{T}}^{\mathrm{miss}}>100 \mathrm{GeV}, M_{\text {eff }}>1000 \mathrm{GeV} .\end{array}$ \\
\hline $2 \gamma$ & $\begin{array}{l}\text { At least two photons with } p_{\mathrm{T}}>60 \mathrm{GeV} \text { and for the leading photon } \\
p_{\mathrm{T}}>90 \mathrm{GeV} . E_{\mathrm{T}}^{\text {miss }}>100 \mathrm{GeV}\end{array}$ \\
\hline $0 \ell+4$ jets & $\begin{array}{l}\text { At least four jets, requiring } p_{\mathrm{T}}>\{50,100\} \mathrm{GeV}, \text { and for the leading jet } \\
p_{\mathrm{T}}>100 \mathrm{GeV} . \text { No leptons with } p_{\mathrm{T}}>10 \mathrm{GeV} . E_{\mathrm{T}}^{\mathrm{miss}}>200 \mathrm{GeV}, \\
M_{\mathrm{eff}}>\{1000,1200\} \mathrm{GeV} \text { and } E_{\mathrm{T}}^{\text {miss }} / M_{\text {eff }}>\{0.2,0.3\} .\end{array}$ \\
\hline $0 \ell+2$ jets & $\begin{array}{l}\text { At least two jets with } p_{\mathrm{T}}>200 \mathrm{GeV} \text {, and for the leading jet } \\
p_{\mathrm{T}}>300 \mathrm{GeV} . \text { No leptons with } p_{\mathrm{T}}>10 \mathrm{GeV} . E_{\mathrm{T}}^{\text {miss }}>400 \mathrm{GeV}, \\
M_{\text {eff }}>1200 \mathrm{GeV} \text { and } E_{\mathrm{T}}^{\text {miss }} / M_{\text {eff }}>0.35 .\end{array}$ \\
\hline
\end{tabular}


the $E_{\mathrm{T}}^{\text {miss }}$ denotes the absolute value of the missing $p_{\mathrm{T}}$, and the effective mass is $M_{\mathrm{eff}}$ defined by

$$
M_{\mathrm{eff}}=E_{\mathrm{T}}^{\mathrm{miss}}+\sum_{j: \text { jet }}^{2 \text { or } 4} p_{\mathrm{T}}(j)+\sum_{\ell: \text { lepton }} p_{\mathrm{T}}(\ell),
$$

where the summation on the jet $p_{\mathrm{T}}$ is for the leading two jets in the two $\tau$-jets mode and the no lepton with two jets mode, and for the leading four jets in other modes. The cuts in each mode are those typically used by the current experiment at the LHC. To fix the numerical values for kinematical variables, we performed simple optimization to maximize the significance. In the four jets with no lepton mode, for which the maximized values depend rather heavily on the location of the model points, we left several cuts unfixed. When a cut has two values in brackets, it means that the kinematical cut is optimized within the described values.

\subsection{Example model points}

We first pick up several example model points to illustrate the result. The 10 selected model points (PT 1 to PT 10) are depicted in Fig. 1 and the corresponding model parameters are summarized in Table 4.

Table 4: Model parameters of the example model points.

\begin{tabular}{|c|c|c|c|c|}
\hline mGMSB & $\Lambda_{s}(\mathrm{TeV})$ & $\Lambda_{g}(\mathrm{TeV})$ & $\tan \beta$ & \\
\hline PT 1 & 350 & 200 & 10 & \\
\hline PT 2 & 350 & 200 & 40 & \\
\hline PT 3 & 150 & 180 & 10 & \\
\hline PT 4 & 150 & 120 & 40 & \\
\hline PT 5 & 100 & 260 & 10 & \\
\hline PT 6 & 120 & 230 & 40 & \\
\hline mSUGRA & $m_{0}(\mathrm{GeV})$ & $m_{1 / 2}(\mathrm{GeV})$ & $\tan \beta$ & $A_{0}$ \\
\hline PT 7 & 260 & 500 & 10 & 0 \\
\hline PT 8 & 260 & 500 & 40 & 0 \\
\hline PT 9 & 1100 & 260 & 10 & 0 \\
\hline PT 10 & 1100 & 260 & 40 & 0 \\
\hline
\end{tabular}

PT 1 to 6 are mGMSB model points. PT 1 and 2 represent models with a Bino-NLSP, 


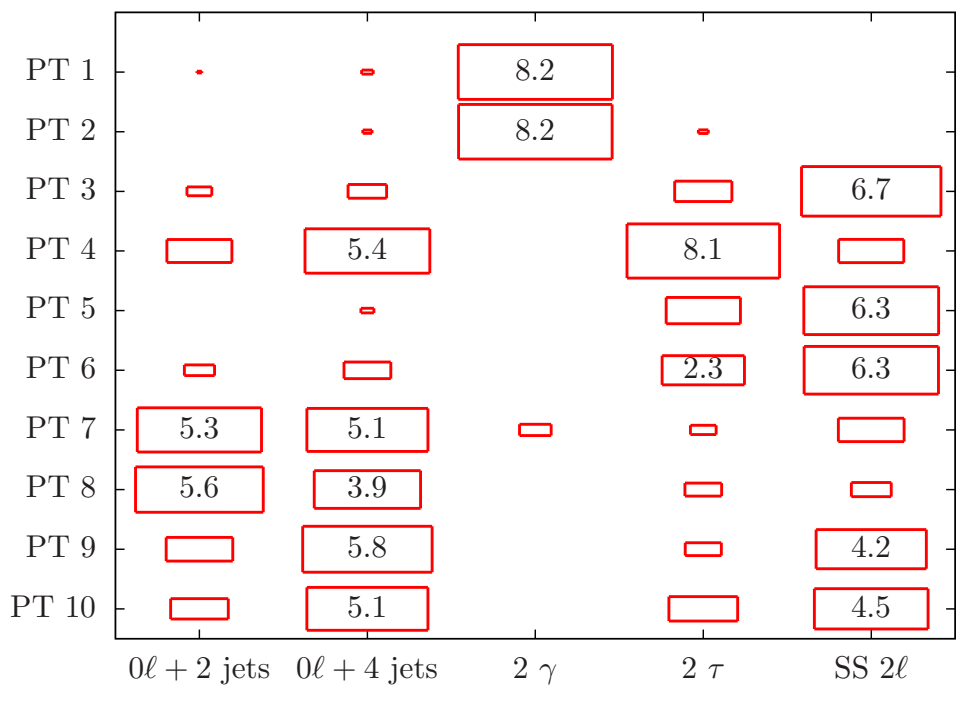

Figure 2: Significances Z's of the example model points for the five modes listed in Tab. 3. The size of red boxes shows the corresponding significance with an integrated luminosity of $30 \mathrm{fb}^{-1}$ at $7 \mathrm{TeV}$ run.

PT 3 and 4 models with a slepton-NLSP but the $\tilde{\tau}_{1}$ mass and the lightest neutralino mass are nearly degenerate, and PT 5 and 6 with a slepton-NLSP without such a degeneracy. PT 7 to 10 are mSUGRA model points. Here, we take $A_{0}=0$. PT 7 and 8 are typical models with small $m_{0}$ and large $m_{1 / 2}$, and PT 9 and 10 are typical models with large $m_{0}$ and small $m_{1 / 2}$. In the Appendix, we list the mass spectrum of relevant superparticles and their dominant decay modes in Tab. 10 and 11.

In Fig. 2, we illustrate the significances of the example model points in the five mode in Tab. 3 with the integrated luminosity of $30 \mathrm{fb}^{-1}$ at $7 \mathrm{TeV}$ run. The number of signal events and our estimation of the SM background is listed in Tab. 12 in the Appendix. We see that the naive expectation in Table 1 is in fact visible in terms of the significances. An exception is PT 4, where the no lepton modes have unexpectedly large significances. At this point, the lighter stau mass is nearly degenerated to the lightest neutralino mass and the significant proportion of taus or leptons from the upper-stream of decay-chains become soft. This fact results in smaller events with high- $p_{\mathrm{T}}$ leptons. 


\subsection{Results for all model points}

We now illustrate results for all model points. Since the significance variable is an increasing function of the number of signal events after the cut, its absolute value scales with the total cross section of the model point. Thus the meaningful quantity for discrimination is the ratio of significances in different modes or a similar quantity. In the following we show the distribution of the significance in each mode in comparison with the one in the four jets with no lepton mode $Z(0 \ell 4 j)$. The significances are calculated for the integrated luminosity of $30 \mathrm{fb}^{-1}$ at $7 \mathrm{TeV}$ run.

We first compare the photon mode significance. Since the multi-photon signal is a very distinctive signature for the mGMSB model with a neutralino-NLSP, the model is expected to be distinguished clearly from the others in terms of the photon mode significance. In Fig. 3(a), we plot the photon mode significance $Z(2 \gamma)$ versus the four jets mode significance $Z(0 \ell 4 j)$. Each point in the figure corresponds to a model point. A red and triangle (blue and circle, black and square) mark represents a mSUGRA (mGMSB with a slepton-NLSP, mGMSB with a neutralino-NLSP) model point. We can check a clear separation of the mGMSB model points with a neutralino-NLSP from the other models as expected. A few model points of the GMSB model with a slepton-NLSP have the significance larger than five. At these model points, the lightest neutralino $\tilde{\chi}_{1}^{0}$ and the lighter slepton are almost degenerated and the decay of the lightest neutralino $\tilde{\chi}_{1}^{0}$ into the lighter slepton is kinematically suppressed. Thus, these model points are parametrically very near to the neutralino-NLSP case and we here treat them as the neutralino-NLSP case.

Next we show the significance in the SS two leptons mode $Z(\mathrm{SS} 2 \ell)$. To clarify the illustration, we deselect the mGMSB model points with a neutralino-NLSP (or more precisely those with $Z(2 \gamma)>3$ ). In Fig. 3(b) (and the following figures), the rest mGMSB model points are marked with blue and circle and the mSUGRA model points are marked with red and triangle. We see a clear inclination that the mSUGRA model points have smaller $Z(\mathrm{SS} 2 \ell) / Z(0 \ell 4 j)$ and the mGMSB model points have larger $Z(\mathrm{SS} 2 \ell) / Z(0 \ell 4 j)$, showing that for most of the model points the lepton richness is indeed a good discriminator even with the smearing due to the SM background and detector effects. However, we also find 


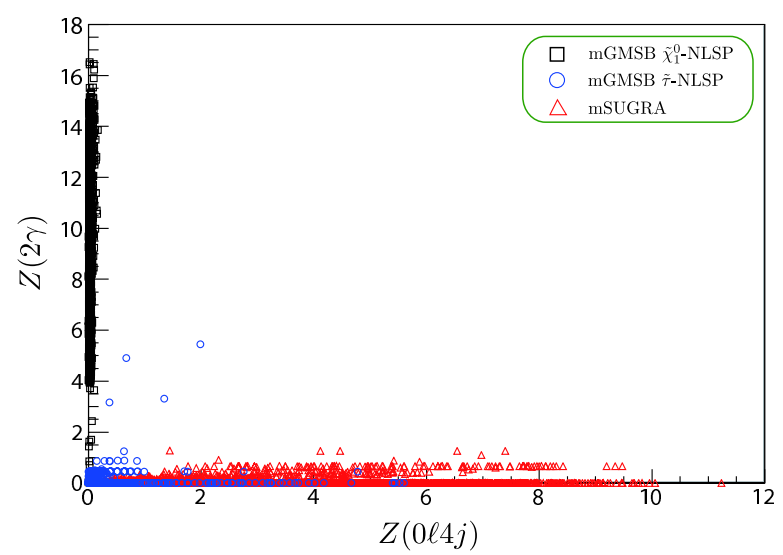

(a) $Z(2 \gamma)$ vs. $Z(0 \ell 4 j)$.

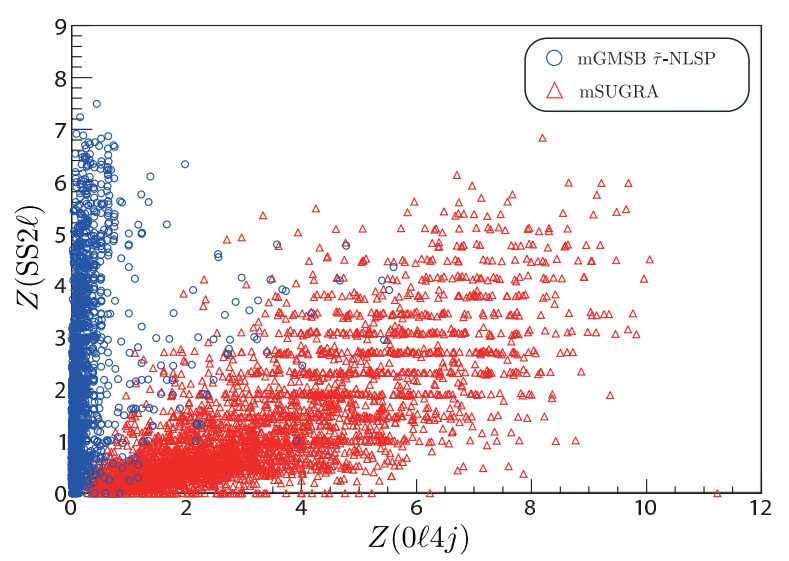

(b) $Z(\mathrm{SS} 2 \ell)$ vs. $Z(0 \ell 4 j)$.

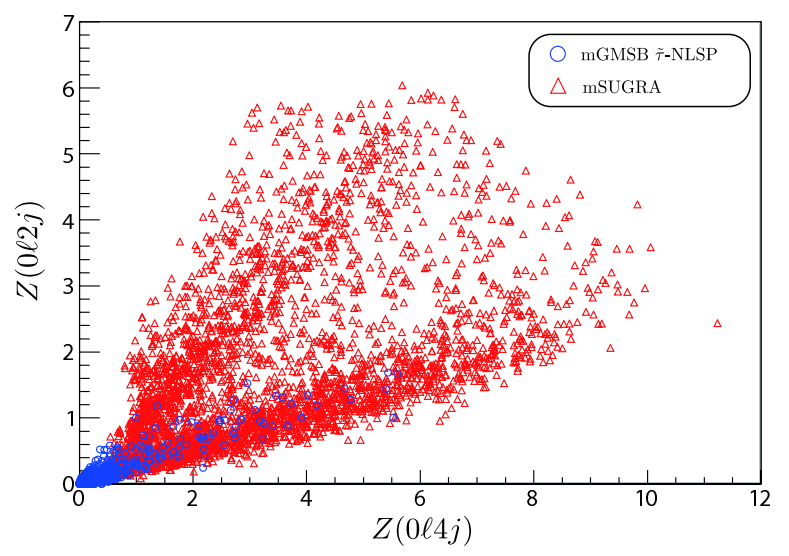

(d) $Z(0 \ell 2 j)$ vs. $Z(0 \ell 4 j)$.

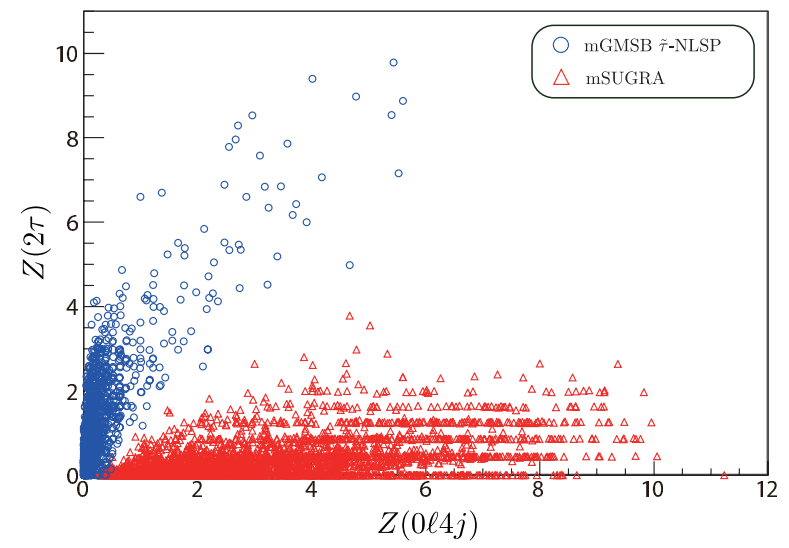

(c) $Z(2 \tau)$ vs. $Z(0 \ell 4 j)$.

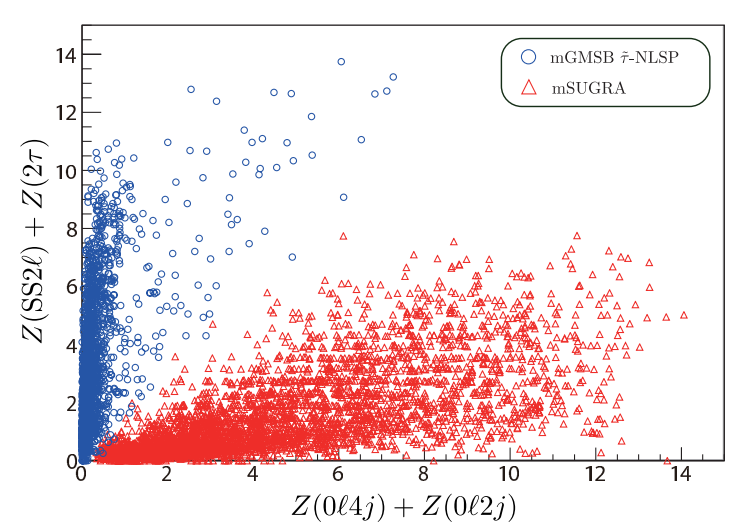

(e) $Z(\mathrm{SS} 2 \ell)+Z(2 \tau)$ vs. $Z(0 \ell 4 j)+Z(0 \ell 2 j)$.

Figure 3: Scatter plots of significances $Z$ 's for an integrated luminosity of $30 \mathrm{fb}^{-1}$ at 7 $\mathrm{TeV}$ run. The black square points are the mGMSB model points with a neutralino-NLSP, the blue circle points the mGMSB model points with a slepton-NLSP, the red triangle points the mSUGRA model points. 
many mGMSB model points with rather small $Z(\mathrm{SS} 2 \ell) / Z(0 \ell 4 j)$. As discussed in the previous section, a typical reason for a smaller $Z(\mathrm{SS} 2 \ell)$ is nearly degenerated NLSPs. If the slepton-NLSP is nearly degenerated to the lightest neutralino, then a possible leptons coming from the decay of the lightest neutralino into the slepton becomes soft and difficult to be detected. Another but related cause for a less lepton signature is larger $\tan \beta$. As indicated in the previous section, when the $\tan \beta$ is large, the SUSY decay chains involve more staus than other sleptons, because large $\tan \beta$ induces the large left-right mixing of the staus, which leads to the small stau mass and large interaction originated from the component of the left-handed stau. Thus decay into the stau is preferred from both reasons of kinematics and interaction strength. Note that the decrease of the leptons results in not only a smaller $Z(\mathrm{SS} 2 \ell)$ but also a larger $Z(0 \ell 4 j)$, showing that a smaller $Z(\mathrm{SS} 2 \ell)$ at those points is not cased by a mere scale of cross section.

To rescue the mGMSB model points with a small lepton mode significance, we now show the significance in the two $\tau$-jets mode versus that in the four jets mode in Fig. 3(c). Here we see an apparently better separation between the mSUGRA and the mGMSB models compared to the dilepton mode. Especially, those mGMSB model points with larger $Z(0 \ell 4 j)$ have larger $Z(2 \tau)$. This is another check that in those model points the leptons are replaced by $\tau$-jets. For the discrimination, this $\tau$-jets mode is important since it plays a complementary role to the dilepton mode. Note that for most of the mGMSB model points, the $Z(2 \tau)$ is small compared to $Z(\operatorname{SS} 2 \ell)$. This is because of the low detection efficiency of the $\tau$-jet.

Finally we look at the two jets mode in Fig. 3(d). The mSUGRA model points are spread over a wide region, but the mGMSB model points have smaller $Z(0 \ell 2 j) / Z(0 \ell 4 j)$. The two jets mode alone does not work as a discriminator, but it strengthen the separation between the mGMSB model points and part of the mSUGRA model points. In summary, with the inclusion of the SM background and detection efficiencies, the photon mode, SS dilepton mode together with two $\tau$-jets mode and their counters, two jets and four jets with no lepton modes, remain serving as discriminator for the mSUGRA and mGMSB models. In Fig. $3(\mathrm{e})$ we plot two sums $Z(\mathrm{SS} 2 \ell)+Z(2 \tau)$ vs. $Z(0 \ell 4 j)+Z(0 \ell 2 j)$ for both models. We see a fairly good separation between the two models.

In reality, the significance values have uncertainties due to the experimental errors and 
errors from the MC statistics. With an increasing integrated luminosity, each model point gains larger absolute values of the significances and thus the distance from the opposite set of models (mSUGRA vs. mGMSB) become larger. Then the next problem is how much the integrated luminosity is needed in order that the model point is well-separated from the opposite models. In the next section, we study the integrated-luminosity dependence of the separation between the two model.

\section{Significance test for discriminating models}

Given the above results, we now go on to the discrimination of the mGMSB and the mSUGRA models. Discrimination here means that excluding the mGMSB models from the mSUGRA models and vice versa. In the previous section, we have seen that even with the inclusion of the effects of the SM background events and the statistical uncertainty, we expect a fairly good separation between the two models at earlier stages of the experiment after the discovery. However, to answer the question of how far and at which luminosity the discrimination is possible we need a further machinery. In this section, we develop a statistical method and answer this question.

The statistical method we use is based on the $\chi^{2}$ test on the significance variables. Given two model points, $m_{a}$ and $m_{b}$, and an integrated luminosity $L$, the $\chi^{2}$ variable is defined as

$$
\chi^{2}\left(m_{a}, m_{b} ; L\right)=\sum_{i: \text { modes }} \frac{\left(Z\left(m_{a}, i ; L\right)-Z\left(m_{b}, i ; L\right)\right)^{2}}{\left(\delta Z\left(m_{a}, i ; L\right)\right)^{2}+\left(\delta Z\left(m_{b}, i ; L\right)\right)^{2}},
$$

where $i$ runs for modes used in the analysis and $\delta Z$ is the error of $Z$. In general, $\delta Z$ contains various systematic errors as well as the statistical error. In the following analysis, we only consider the expected experimental statistical error and the uncertainty from MC statistics. We may also define $\chi^{2}$ by using functions of significance variables. Then the denominator of Eq. (10) is replaced by the error of corresponding functions.

If, for the two model points, the calculated $\chi^{2}$ is smaller than a certain value, their difference of significances are considered as statistically insignificant. In the following analysis, we use a $p$-value of $95 \%$ for this criterion. The corresponding $\chi^{2}$ value depends on the number of degrees of freedom, i.e. the number of significances used for the calculation 
of $\chi^{2}$. We say that two model points are in the vicinity of each other if their $\chi^{2}$ is smaller than than this value.

Now, we consider the problem of testing whether a certain model $m_{0}$ is consistent as an mGMSB model or an mSUGRA model. We first prepare a set of model points in these models. We denote by $M$ the set of model points and indicate as $M=G$ the set of mGMSB model points and $M=S$ that of the mSUGRA models, respectively. Then let us perform a $\chi^{2}$ test and collect those model points of $M$ in the vicinity of $m_{0}$. We denote this set of model points as $S_{M}\left(m_{0}\right)$. If $S_{M}\left(m_{0}\right)$ is not empty, there is a certain probability that $m_{0}$ is in the model of $M$. However, we must take account of the density of points in $M$. If there are a large number of points in $M$, there might be a high probability of the $S_{M}\left(m_{0}\right)$ being non-empty even if $m_{0}$ is not a good candidate in the model of $M$. On the other hand, if the cardinality of $M$ is small there may be a possibility that $S_{M}\left(m_{0}\right)$ is empty even if $m_{0}$ is actually in the model of $M$.

For this purpose, we first consider a point $m$ in $M$ as $m_{0}$. For the care of this last problem, we must prepare a dense enough set $M$. Then for each point $m$ in $M, S_{M}(m)$ contains at least a few points. The number of points in $S_{M}(m)$ indicates the sensitivity of the significances in the vicinity of $m$. We now consider a quantity $s_{M}(m)$ defined as

$$
s_{M}(m)=\sum_{m^{\prime} \in S_{M}(m)} \frac{1}{\# S_{M}\left(m^{\prime}\right)} .
$$

If the variation of $\# S_{M}(m)$ is smooth compared to the density of model points around $m$ in the region of $S_{M}(m)$, the value of $s_{M}(m)$ is near unity. Similarly, we define for a general model point $m_{0}$

$$
s_{M}\left(m_{0}\right)=\sum_{m^{\prime} \in S_{M}\left(m_{0}\right)} \frac{1}{\# S_{M}\left(m^{\prime}\right)} .
$$

If the density of $M$ is high enough and the variation of $\# S_{M}(m)$ is smooth, we expect that $s_{M}\left(m_{0}\right)$ is also near unity if $m_{0}$ is indeed in the model of $M$. Note that this condition is stronger than the mere condition that $S_{M}\left(m_{0}\right)$ is not empty. In this method of discrimination, the condition for $m_{0}$ to be consistent with being in the model of $M$ is that $s_{M}\left(m_{0}\right)$ is near to unity within the errors, which are discussed further below.

Before discussing the errors, we have several remarks on this method. Let us consider the limit of infinite density of $M$ and infinite luminosity. Then the distribution of model 
points in $M$ in the significance space spreads over a certain region and then $s_{M}$ for a given model is unity or zero, indicating the model is in or out of this region. In other words, this method is a sort of in-or-out test and does not take the density distribution of $M$ in this limit. So this method is useful to discriminate well separated models, but may be useless for those models, in which a tiny amount of model points spreads over a wide region. In practice, we have a finite density of $M$ and a finite luminosity, and the region is approximated by discrete points. In this case, the sufficiency of the density of $M$ may be tested by $S_{M}(m)$ for $m$ in $M$ as noted above. There may be some points with a small $S_{M}(m)$ compared to the rest. One reason for such a case is the existence of points, called "cliffs" in Ref. [24]. Around these points the variation of the significance is large compared to that of the parameter. The boundaries in the parameter space or in the mass spectrum space may be a cause for such a case. This case is often physically interesting and may require further and detailed studies.

The errors come from the effects of both the finite density and luminosity. In a case of finite luminosity, we have a certain variation of the value of the $s_{M}\left(m_{0}\right)$ other than unity or zero if $m_{0}$ is near the boundary of $M$ in the significance space. Assuming $M$ is uniformly distributed, the $s_{M}\left(m_{0}\right)$ at the boundary is equal to $\log 2 \simeq 0.69$ (independent of the dimension of the significances) and increases as $m_{0}$ moves from the boundary into the region of $M$ to a maximal of $\sim 1.2$ depending on the dimension. Away from the boundary $s_{M}\left(m_{0}\right)$ decreases to zero. These values may vary well according to the shape and distribution of concerning model $M$. Since we do not know the distribution of $M$ and the position of $m_{0}$ in the significance space a priori, we handle this variation as an uncertainty and we take the value $\delta_{l} s_{M}\left(m_{0}\right)=0.5$ for this uncertainty in the following analysis. There are also errors on $s_{M}\left(m_{0}\right)$ coming from the finite density of $M$. We adopt a rough approximation of this error by assuming that $S_{M}\left(m_{0}\right)$ shapes like a multidimensional sphere in the space of significances. In this approximation, the error of $s_{M}\left(m_{0}\right)$ is given by

$$
\delta_{g} s_{M}\left(m_{0}\right)=s_{M}\left(m_{0}\right) d\left(\frac{C_{d}}{\# S_{M}\left(m_{0}\right)}\right)^{1 / d},
$$

where $d$ is the degrees of freedom of $\chi^{2}$ and $C_{d}=\pi^{d / 2} / \Gamma\left(\frac{d}{2}+1\right)$. As a result, the condition 
for the consistency of $m_{0}$ in the model $M$ can be written as

$$
\left|s_{M}\left(m_{0}\right)-1\right|<\delta s_{M}\left(m_{0}\right)=\sqrt{\delta_{g} s_{M}\left(m_{0}\right)^{2}+\delta_{l} s_{M}\left(m_{0}\right)^{2}} .
$$

We remark that this estimation of $\delta s_{M}$ is very rough and we assume that the variation of $S_{M}$ in the parameter space is smooth.

We examine this method of model discrimination with the result of the Monte Carlo simulation in Sec. 3.7. We use as the set $M=G$ or $S$ the set of all simulated model points described in Sec. 3.4. As test model points, we choose from the same set model points which pass the experimental constraints and satisfy the discovery condition, for which we require at least one of the significances is greater than five for $30 \mathrm{fb}^{-1}$. The set of test model points are denoted by $G^{\prime}$ and $S^{\prime}$. The number of model points are 4046, 1091, 5633 and 754 for $G, G^{\prime}, S$ and $S^{\prime}$, respectively. We show the results for $\chi^{2}$ calculated in three different combinations of the modes listed in Table 3. The first one uses the 0 lepton 4 jets mode, 2 photons mode and SS 2 leptons mode, the second one uses the sum of two 0 lepton modes, 2 photons mode and the sum of $2 \tau$-jets and 2 leptons modes, and the third one uses all the five modes. We call these three cases as T1 to T3. The results are illustrated with integrated luminosities $L=1,3,10,30$ and $100 \mathrm{fb}^{-1}$ in the $7 \mathrm{TeV}$ run.

We first check model points with small \# $S_{M}$. In Table 5 , the number of model points in $M=G$ and $S$ with \# $S_{M}$ less than ten is presented. Because most of the points with

Table 5: The number of model points with $\# S_{M}<10$, which pass the experimental constraints. The total number of model points are 3048 for $G$ and 3654 for $S$. The numbers for $L=1$ and $3 \mathrm{fb}^{-1}$ are all zero.

\begin{tabular}{|c|c|c|c|}
\hline$L$ & $10 \mathrm{fb}^{-1}$ & $30 \mathrm{fb}^{-1}$ & $100 \mathrm{fb}^{-1}$ \\
\hline \hline$G$ in T1 & 0 & 1 & 2 \\
\hline$G$ in T2 & 1 & 3 & 3 \\
\hline$G$ in T3 & 0 & 2 & 4 \\
\hline \hline$S$ in T1 & 0 & 0 & 0 \\
\hline$S$ in T2 & 0 & 0 & 0 \\
\hline$S$ in T3 & 0 & 0 & 0 \\
\hline
\end{tabular}

$\# S_{M}<10$ are near the artificial boundary in the model parameter space, especially in the 
low mass region, and they are unlikely to affect the result of the test, only those which pass the experimental constraints are counted in the table. The few mGMSB model points in the table are those nearly on the boundary where the NLSP changes. The vicinity of these points is a good example of a "cliff". The followed result shows the effect of this region for discriminating the models, but a further study might be important if the experiment reveals its possibility.

Now we present our result of the tests T1 to T3. In Table 6, $s_{G}$ and $s_{S}$ averaged in $G^{\prime}$ or $S^{\prime}$ are presented. First, we see that $s_{M}$ calculated in the corresponding model

Table 6: Evolution of the averaged $s_{G, S}$ with respect to the integrated luminosity $L$ for significance test T1 to T3.

\begin{tabular}{|c|c|c|c|c|c|c|}
\hline & $L$ & $1 \mathrm{fb}^{-1}$ & $3 \mathrm{fb}^{-1}$ & $10 \mathrm{fb}^{-1}$ & $30 \mathrm{fb}^{-1}$ & $100 \mathrm{fb}^{-1}$ \\
\hline \hline T1 & $s_{G}$ averaged in $G^{\prime}$ & 1.036 & 1.042 & 1.011 & 1.009 & 0.99 \\
\hline & $s_{S}$ averaged in $G^{\prime}$ & 0.8768 & 0.5584 & 0.1107 & 0.0412 & 0.034 \\
\hline & $s_{G}$ averaged in $S^{\prime}$ & 1.119 & 0.7287 & 0.4493 & 0.4751 & 0.4448 \\
\hline & $s_{S}$ averaged in $S^{\prime}$ & 1.062 & 1.202 & 1.009 & 0.9293 & 0.9002 \\
\hline \hline T2 & $s_{G}$ averaged in $G^{\prime}$ & 1.031 & 1.033 & 1.01 & 1.019 & 0.997 \\
\hline & $s_{S}$ averaged in $G^{\prime}$ & 0.823 & 0.5146 & 0.0842 & 0.0388 & 0.0377 \\
\hline & $s_{G}$ averaged in $S^{\prime}$ & 0.911 & 0.2632 & 0.0274 & 0.0344 & 0.0041 \\
\hline & $s_{S}$ averaged in $S^{\prime}$ & 1.158 & 1.201 & 1.072 & 1.049 & 0.992 \\
\hline \hline T3 & $s_{G}$ averaged in $G^{\prime}$ & 1.03 & 1.057 & 0.9868 & 1.019 & 1.009 \\
\hline & $s_{S}$ averaged in $G^{\prime}$ & 0.869 & 0.6141 & 0.1164 & 0.0085 & 0.0067 \\
\hline & $s_{G}$ averaged in $S^{\prime}$ & 1.001 & 0.428 & 0.0247 & 0.0034 & 0.0003 \\
\hline & $s_{S}$ averaged in $S^{\prime}$ & 1.101 & 1.177 & 0.947 & 0.8359 & 0.783 \\
\hline
\end{tabular}

itself is consistent with unity. There seems a tendency of slightly lower values in the high luminosity especially for the mSUGRA case in T3. This may be due to varying errors of the significances by the statistical uncertainty of the Monte Carlo simulation. We checked this tendency vanishes if we exclude this uncertainty in the error of the significances. (See Table 8.) In contrast, $s_{M}$ calculated in the wrong model decreases with higher integrated luminosity, indicating that more model points are discriminated from the other model.

This fact is more clear in Table 7, where the number of falsified model points using the criterion in Eq. (14) is presented. We see a large portion of the model points are falsified 
Table 7: The number of falsified model points with respect to the integrated luminosity L. Eq. (14) is used for the falsification criteria.

\begin{tabular}{|c|c|c|c|c|c|c|}
\hline & $L$ & $1 \mathrm{fb}^{-1}$ & $3 \mathrm{fb}^{-1}$ & $10 \mathrm{fb}^{-1}$ & $30 \mathrm{fb}^{-1}$ & $100 \mathrm{fb}^{-1}$ \\
\hline$\overline{\mathrm{T} 1}$ & $G^{\prime}$ pts $(/ 1091)$ falsified in $G$ & 0 & 1 & 2 & 1 & 4 \\
\hline & $G^{\prime}$ pts $(/ 1091)$ falsified in $S$ & 0 & 323 & 994 & 1051 & 1058 \\
\hline & $S^{\prime}$ pts $(/ 754)$ falsified in $G$ & 0 & 126 & 199 & 176 & 169 \\
\hline & $S^{\prime}$ pts $(/ 754)$ falsified in $S$ & 0 & 0 & 0 & 5 & 6 \\
\hline$\overline{\mathrm{T} 2}$ & "G pts (/1091) falsified in $G$ & $\overline{0}$ & 1 & 2 & 0 & 0 \\
\hline & $G^{\prime}$ pts $(/ 1091)$ falsified in $S$ & 0 & 394 & 1049 & 1089 & 1089 \\
\hline & $S^{\prime}$ pts $(/ 754)$ falsified in $G$ & 10 & 523 & 753 & 753 & 751 \\
\hline & $S^{\prime}$ pts $(/ 754)$ falsified in $S$ & 0 & 0 & 0 & 0 & 0 \\
\hline$\overline{\mathrm{T} 3}$ & 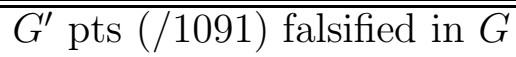 & $\overline{0}$ & $\overline{0}$ & $\overline{1}$ & $\overline{0}$ & $\overline{0}$ \\
\hline & $G^{\prime}$ pts $(/ 1091)$ falsified in $S$ & 0 & 136 & 921 & 1091 & 1091 \\
\hline & $S^{\prime}$ pts $(/ 754)$ falsified in $G$ & 0 & 336 & 752 & 753 & 754 \\
\hline & $S^{\prime}$ pts $(/ 754)$ falsified in $S$ & 0 & 0 & 0 & 1 & 1 \\
\hline
\end{tabular}

from the other model at higher luminosity while most of them remain consistent within the home model. Let us see each tests in more detail. In T1, many mSUGRA model points remain consistent with being a mGMSB model even at $100 \mathrm{fb}^{-1}$. This is because without using the tau-jet mode, the slepton-NLSP GMSB model with nearly degenerated stau and the lightest neutralino may not be discriminated from mSUGRA models as described in the previous section. These mGMSB model points conversely remain unfalsified as a mSUGRA model. These models may be discriminated by using all five modes as in T3. The result shows all mGMSB model points are falsified from being in the mSUGRA model set at $30 \mathrm{fb}^{-1}$. The T2 using combined significances also shows a good separation of models. In particular, it shows a better falsifiability in lower luminosity. This is because T2 uses only three variables for the $\chi^{2}$ test and has an advantage in statistics. In Table 8 , we list the same result but without the Monte Carlo statistical error in the significances for T3. An important conclusion of this result is that even with the inclusion of the SM background and the effect of the detector efficiencies, the model discrimination among the GMSB and SUGRA models using the method gives a very good result at the $30 \mathrm{fb}^{-1}$, i.e. at the time of the $5 \sigma$ (or more) discovery. 
Table 8: Same as Tables 6 and 7 for T3, without the Monte Carlo statistical error included in the significances.

\begin{tabular}{|c|c|c|c|c|c|c|}
\hline & $L$ & $1 \mathrm{fb}^{-1}$ & $3 \mathrm{fb}^{-1}$ & $10 \mathrm{fb}^{-1}$ & $30 \mathrm{fb}^{-1}$ & $100 \mathrm{fb}^{-1}$ \\
\hline \hline T3 & $s_{G}$ averaged in $G^{\prime}$ & 1.031 & 1.053 & 0.974 & 1.009 & 1.012 \\
\hline & $s_{S}$ averaged in $G^{\prime}$ & 0.856 & 0.631 & 0.093 & 0.007 & 0 \\
\hline & $s_{G}$ averaged in $S^{\prime}$ & 0.996 & 0.394 & 0.014 & 0 & 0 \\
\hline & $s_{S}$ averaged in $S^{\prime}$ & 1.153 & 1.277 & 1.06 & 1.041 & 0.997 \\
\hline \hline & $G^{\prime}$ pts $(/ 1091)$ falsified in $G$ & 0 & 0 & 1 & 0 & 0 \\
\hline & $G^{\prime}$ pts $(/ 1091)$ falsified in $S$ & 0 & 137 & 959 & 1091 & 1091 \\
\hline & $S^{\prime}$ pts $(/ 754)$ falsified in $G$ & 0 & 360 & 752 & 754 & 754 \\
\hline & $S^{\prime}$ pts $(/ 754)$ falsified in $S$ & 0 & 0 & 0 & 0 & 0 \\
\hline
\end{tabular}

In the above result, the model points are generated on a flat grid in the parameter space and the tested models $G^{\prime}$ and $S^{\prime}$ are subset of $G$ and $S$, respectively. There is a worry that the number of mis-falsified models is underestimated and there might exist small characteristic regions dropped out from the grid. To check this problem, we generate a new set of test model points randomly in the parameter space. The used regions in the parameter space is $-100 \leq \Lambda_{s} \leq 400(\mathrm{TeV}), 100 \leq \Lambda_{g} \leq 400(\mathrm{TeV})$ and $5 \leq$ $\tan \beta \leq 50$ for the mGMSB model and $0 \leq m_{0} \leq 1200(\mathrm{GeV}), 100 \leq m_{1 / 2} \leq 600(\mathrm{GeV})$, $5 \leq \tan \beta \leq 50$ and $-1 \leq A_{0} / m_{0} \leq 1$ for the mSUGRA model. After requiring the experimental constraints and the discovery condition $\left(5 \sigma\right.$ at $\left.30 \mathrm{fb}^{-1}\right)$, we generated 885 model points (denoted by $G^{\prime \prime}$ ) for the mGMSB model and 1131 model points (denoted by $S^{\prime \prime}$ ) for the mSUGRA model. We present the result of the discrimination test T3 in Table 9. The result is essentially same as the previous result for $G^{\prime}$ and $S^{\prime}$ and showing

Table 9: The number of falsified model points by the significance test T3 for randomly chosen model points in $G^{\prime \prime}$ and $S^{\prime \prime}$.

\begin{tabular}{|c|c|c|c|c|c|c|}
\hline & $L$ & $1 \mathrm{fb}^{-1}$ & $3 \mathrm{fb}^{-1}$ & $10 \mathrm{fb}^{-1}$ & $30 \mathrm{fb}^{-1}$ & $100 \mathrm{fb}^{-1}$ \\
\hline T3 & $G^{\prime \prime} \operatorname{pts}(/ 885)$ falsified in $G$ & 0 & 3 & 4 & 4 & 5 \\
\hline & $G^{\prime \prime} \operatorname{pts}(/ 885)$ falsified in $S$ & 0 & 133 & 809 & 885 & 885 \\
\hline & $S^{\prime \prime} \operatorname{pts}(/ 1131)$ falsified in $G$ & 0 & 507 & 1129 & 1131 & 1131 \\
\hline & $S^{\prime \prime} \operatorname{pts}(/ 1131)$ falsified in $S$ & 0 & 0 & 0 & 0 & 0 \\
\hline
\end{tabular}


the underestimation is very small.

\section{Discussion and conclusion}

In this paper, we discussed a strategy of model discrimination between the mSUGRA and the mGMSB models at a very early stage of the LHC by comparing significances in multiple search modes. We defined the difference of the LHC signatures among model points in a quantitative form and show that the discrimination is possible with a relatively low integrated luminosity. Our estimation of the significances is conservative and the discrimination may be better in the actual experiments. This model discrimination is very important for both model-building and cosmology, since they predict completely different cosmological history and the dark matter. We used a relatively small number of modes in this paper. Although such a coarse graining of LHC signals gives only coarse information, it can discriminate the models qualitatively and guides to research at a much higher integrated luminosity. Though in this paper, we focused on only discrimination between the mSUGRA and mGMSB models, our method can be applied for more generic cases. For example, we can also discuss the discrimination within the mSUGRA models. The so-called co-annihlation and focus regions, which are favored from the viewpoint of dark matter, can be separated by using similar method. This method makes it possible to compare the LHC signals of generic BSM models qualitatively.

\section{Acknowledgement}

We would like to thank K. Hamaguchi and T. T. Yanagida for useful discussions. The work of E.N. is supported in part by JSPS Research Fellowships for Young Scientists.

\section{A Supplemental data of example model points}

In this appendix, we collect supplemental data of our example model points in Tab. 4 for convenience. In Tab. 10 and 11, we list up the mass spectrum of the superparticles of the example model points together with their dominant decay channels.

In Tab. 12, we list the number of signal events for the example model points and the 
Table 10: Superparticles' masses (in unit of $\mathrm{GeV}$ ) and their dominant decay channels for the example model points PT 1 to 6 (mGMSB model) in Tab. 4. From top to bottom, the particles' label represent gluino, down-type squarks, stop, sbottom, selectron, stau, charginos and neutralinos. The gravitino is denoted by $\tilde{G}$.

\begin{tabular}{|c|c|c|c|c|c|c|}
\hline & $\overline{\mathrm{PT} 1}$ & Decay mode & $\overline{\mathrm{PT} 2}$ & Decay mode & PT 3 & Decay mode \\
\hline$\tilde{g}$ & 1530.7 & $\tilde{\chi}_{1}^{ \pm} q \bar{q}^{\prime}, \tilde{\chi}_{2}^{0} q \bar{q}, \tilde{\chi}_{1}^{0} q \bar{q}$ & 1530.1 & $\tilde{\chi}_{1}^{ \pm} q \bar{q}^{\prime}, \tilde{\chi}_{2}^{0} q \bar{q}, \tilde{\chi}_{1}^{0} q \bar{q}$ & 1319.4 & $\tilde{q} q$ \\
\hline$\tilde{d}_{L}$ & 2546.9 & $\tilde{g} d, \tilde{\chi}_{1}^{-} u$ & 2546.3 & $\tilde{g} d, \tilde{\chi}_{1}^{-} u$ & 1306.4 & $\tilde{\chi}_{1}^{-} u, \tilde{\chi}_{2}^{-} u$ \\
\hline$\tilde{d}_{R}$ & 2414.7 & $\tilde{g} d$ & 2414.0 & $\tilde{g} d$ & 1252.4 & $\tilde{\chi}_{1}^{0} d$ \\
\hline$\tilde{t}_{2}$ & 2472.2 & $\tilde{g} t, \tilde{\chi}_{1}^{+} b$ & 2441.1 & $\tilde{g} t, \tilde{\chi}_{1}^{+} b$ & 1277.2 & $\tilde{\chi}_{4}^{0} t, \tilde{\chi}_{3}^{0} t, \tilde{\chi}_{2}^{+} b$ \\
\hline$\tilde{t}_{1}$ & 2208.4 & $\tilde{g} t, \tilde{\chi}_{2}^{+} b$ & 2210.9 & $\tilde{g} t, \tilde{\chi}_{2}^{+} b$ & 1147.8 & $\tilde{\chi}_{1}^{+} b, \tilde{\chi}_{3}^{0} t, \tilde{\chi}_{2}^{+} b$ \\
\hline$\tilde{b}_{2}$ & 2454.8 & $\tilde{g} b, \tilde{\chi}_{2}^{-} t$ & 2428.0 & $\tilde{g} b, \tilde{\chi}_{2}^{-} t$ & 1261.0 & $\tilde{\chi}_{2}^{-} t, \tilde{\chi}_{1}^{-} t$ \\
\hline$\tilde{b}_{1}$ & 2409.2 & $\tilde{g} b$ & 2344.6 & $\tilde{g} b$ & 1247.0 & $\tilde{\chi}_{1}^{-} t, \tilde{\chi}_{2}^{-} t$ \\
\hline$\tilde{e}_{L}$ & 851.1 & $\tilde{\chi}_{1}^{-} \nu, \tilde{\chi}_{2}^{0} e, \tilde{\chi}_{1}^{0} e$ & 850.7 & $\tilde{\chi}_{1}^{-} \nu, \tilde{\chi}_{2}^{0} e, \tilde{\chi}_{1}^{0} e$ & 394.4 & $\tilde{\chi}_{1}^{0} e$ \\
\hline$\tilde{e}_{R}$ & 415.7 & $\tilde{\chi}_{1}^{0} e$ & 416.9 & $\tilde{\chi}_{1}^{0} e$ & 188.7 & $\tilde{G} e$ \\
\hline$\tilde{\tau}_{2}$ & 848.2 & $\tilde{\chi}_{1}^{-} \nu, \tilde{\chi}_{2}^{0} \tau, \tilde{\chi}_{1}^{0} \tau$ & 841.8 & $\tilde{\chi}_{1}^{-} \nu, \tilde{\chi}_{2}^{0} \tau, \tilde{\chi}_{1}^{0} \tau$ & 392.1 & $\tilde{\chi}_{1}^{0} \tau$ \\
\hline$\tilde{\tau}_{1}$ & 422.0 & $\tilde{\chi}_{1}^{0} \tau$ & 367.4 & $\tilde{\chi}_{1}^{0} \tau$ & 191.3 & $\tilde{G} \tau$ \\
\hline$\tilde{\chi}_{2}^{+}$ & 877.7 & $\tilde{\chi}_{1}^{+} h / Z, \tilde{\chi}_{2}^{0} W^{+}$ & 860.8 & $\tilde{\chi}_{1}^{+} h / Z, \tilde{\chi}_{2}^{0} W^{+}$ & 537.7 & $\tilde{\ell} \nu, \tilde{\nu} \ell$ \\
\hline$\tilde{\chi}_{1}^{+}$ & 529.8 & $\tilde{\chi}_{1}^{0} W^{+}$ & 531.3 & $\tilde{\tau} \nu, \tilde{\chi}_{1}^{0} W^{+}$ & 423.8 & $\tilde{\chi}_{1}^{0} W^{+}, \tilde{\nu} \ell$ \\
\hline$\tilde{\chi}_{4}^{0}$ & 877.3 & $\tilde{\chi}_{1}^{+} W^{-}, \tilde{\chi}_{2}^{0} h$ & 860.2 & $\tilde{\chi}_{1}^{+} W^{-}, \tilde{\chi}_{2}^{0} h$ & 539.8 & $\tilde{\ell} \ell, \tilde{\nu} \nu$ \\
\hline$\tilde{\chi}_{3}^{0}$ & 867.6 & $\tilde{\chi}_{1}^{+} W^{-}, \tilde{\chi}_{2}^{0} Z$ & 852.0 & $\tilde{\chi}_{1}^{+} W^{-}, \tilde{\chi}_{2}^{0} Z$ & 483.0 & $\tilde{\chi}_{1}^{0} Z, \tilde{\ell} \ell$ \\
\hline$\tilde{\chi}_{2}^{0}$ & 529.5 & $\tilde{\chi}_{1}^{0} h$ & 530.8 & $\tilde{\tau} \tau, \tilde{\chi}_{1}^{0} h$ & 422.2 & $\tilde{\chi}_{1}^{0} h, \tilde{\ell} \ell$ \\
\hline$\tilde{\chi}_{1}^{0}$ & 273.4 & $\tilde{G} \gamma / Z$ & 273.8 & $\tilde{G} \gamma / Z$ & 240.6 & $\tilde{\ell}_{R} \ell, \tilde{\tau}_{1} \tau$ \\
\hline & $\overline{\overline{\mathrm{PT}} 4}$ & "Decay mode & $\overline{\overline{\text { PT } 5}}$ & "Decay mode & PT 6 & "Decay mode \\
\hline$\tilde{g}$ & 941.5 & $\tilde{\chi}_{2}^{0} q \bar{q}, \tilde{\chi}_{1}^{0} q \bar{q}, \tilde{\chi}_{1}^{ \pm} q \bar{q}^{\prime}$ & 1770.1 & $\tilde{q} q$ & 1595.6 & $\tilde{q} q$ \\
\hline$\tilde{d}_{L}$ & 1192.3 & $\tilde{g} d, \tilde{\chi}_{1}^{-} u$ & 1253.0 & $\tilde{\chi}_{2}^{-} u, \tilde{\chi}_{4}^{0} d$ & 1262.1 & $\tilde{\chi}_{2}^{-} u, \tilde{\chi}_{4}^{0} d$ \\
\hline$\tilde{d}_{R}$ & 1137.5 & $\tilde{g} d$ & 1218.1 & $\tilde{\chi}_{1}^{0} d$ & 1220.2 & $\tilde{\chi}_{1}^{0} d$ \\
\hline$\tilde{t}_{2}$ & 1146.5 & $\tilde{\chi}_{1}^{+} b, \tilde{\chi}_{4}^{0} t, \tilde{\chi}_{3}^{0} t$ & 1242.6 & $\tilde{\chi}_{3}^{0} t, \tilde{\chi}_{2}^{0} t, \tilde{\chi}_{2}^{+} b$ & 1230.4 & $\tilde{\chi}_{2}^{+} b, \tilde{\chi}_{3}^{0} t, \tilde{\chi}_{2}^{0} t$ \\
\hline$\tilde{t}_{1}$ & 1042.6 & $\tilde{\chi}_{2}^{+} b, \tilde{\chi}_{3}^{0} t$ & 1120.1 & $\tilde{\chi}_{1}^{+} b, \tilde{\chi}_{3}^{0} t, \tilde{\chi}_{2}^{0} t$ & 1122.6 & $\tilde{\chi}_{1}^{+} b, \tilde{\chi}_{3}^{0} t, \tilde{\chi}_{2}^{0} t$ \\
\hline$\tilde{b}_{2}$ & 1139.4 & $\tilde{\chi}_{2}^{-} t, \tilde{g} b$ & 1220.5 & $\tilde{\chi}_{1}^{-} t, \tilde{\chi}_{2}^{-} t$ & 1218.6 & $\tilde{\chi}_{1}^{-} t, \tilde{\chi}_{2}^{-} t$ \\
\hline$\tilde{b}_{1}$ & 1093.5 & $\tilde{g} b, \tilde{\chi}_{1}^{-} t$ & 1209.5 & $\tilde{\chi}_{1}^{-} t, \tilde{\chi}_{2}^{-} t$ & 1178.0 & $\tilde{\chi}_{1}^{-} t, \tilde{\chi}_{2}^{0} b$ \\
\hline$\tilde{e}_{L}$ & 378.7 & $\tilde{\chi}_{1}^{-} \nu, \tilde{\chi}_{1}^{0} e, \tilde{\chi}_{2}^{0} e$ & 327.3 & $\tilde{\tau}_{1} \tau e$ & 350.1 & $\tilde{\chi}_{1}^{0} e$ \\
\hline$\tilde{e}_{R}$ & 184.9 & $\tilde{\chi}_{1}^{0} e$ & 146.7 & $\tilde{G} e$ & 163.0 & $\tilde{\tau} \tau e$ \\
\hline$\tilde{\tau}_{2}$ & 383.5 & $\tilde{\tau}_{1} Z / h, \tilde{\chi}_{1}^{0} \tau$ & 322.3 & $\tilde{\tau}_{1} Z / h$ & 354.9 & $\tilde{\tau}_{1} Z / h$ \\
\hline$\tilde{\tau}_{1}$ & 137.5 & $\tilde{G} \tau$ & 149.5 & $\tilde{G} \tau$ & 114.1 & $\tilde{G} \tau$ \\
\hline$\tilde{\chi}_{2}^{+}$ & 473.0 & $\tilde{\chi}_{2}^{0} W^{+}, \tilde{\chi}_{1}^{+} Z$ & 688.2 & $\tilde{\ell} \nu, \tilde{\nu} \ell$ & 616.8 & $\tilde{\ell} \nu, \tilde{\nu} \ell$ \\
\hline$\tilde{\chi}_{1}^{+}$ & 304.8 & $\tilde{\tau} \nu$ & 445.2 & $\tilde{\nu} \ell, \tilde{\chi}_{1}^{0} W^{+}$ & 441.5 & $\tilde{\tau}_{1} \nu, \tilde{\nu} \tau$ \\
\hline$\tilde{\chi}_{4}^{0}$ & 472.9 & $\tilde{\chi}_{1}^{+} W^{-}, \tilde{\chi}_{2}^{0} h$ & 695.3 & $\tilde{\nu} \nu, \tilde{\ell}_{L} \ell$ & 623.1 & $\tilde{\ell} \ell, \tilde{\nu} \nu$ \\
\hline$\tilde{\chi}_{3}^{0}$ & 453.7 & $\tilde{\chi}_{1}^{+} W^{-}, \tilde{\tau}_{1} \tau, \tilde{\chi}_{2}^{0} Z$ & 454.0 & $\tilde{\chi}_{1}^{0} Z, \tilde{\tau}_{1} \tau$ & 454.6 & $\tilde{\tau}_{1} \tau$ \\
\hline$\tilde{\chi}_{2}^{0}$ & 304.1 & $\tilde{\tau}_{1} \tau$ & 447.3 & $\tilde{\tau}_{1} \tau, \tilde{\ell}_{R} \ell$ & 439.4 & $\tilde{\tau}_{1} \tau$ \\
\hline$\tilde{\chi}_{1}^{0}$ & 160.9 & $\tilde{\tau}_{1} \tau$ & 342.8 & $\tilde{\tau}_{1} \tau, \tilde{\ell}_{R} \ell$ & 306.3 & $\tilde{\tau}_{1} \tau, \tilde{\ell}_{R} \ell$ \\
\hline
\end{tabular}


Table 11: Same as Tab. 10, but for example model points PT 7 to 10 (mSUGRA model) in Tab. 4. Since the list of decay modes is very similar for PT 9 and PT 10, their lists are gathered.

\begin{tabular}{|c|c|c|c|c|c|c|c|}
\hline & $\overline{\mathrm{PT} 7}$ & 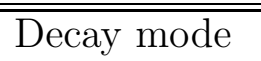 & $\overline{\overline{\mathrm{PT}} 8}$ & 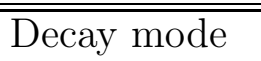 & $\overline{\overline{\text { PT } 9}}$ & PT 10 & 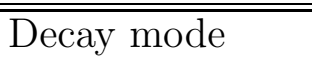 \\
\hline$\tilde{g}$ & 1157.2 & $\tilde{q} q$ & 1156.7 & $\tilde{q} q$ & 689.7 & 689.1 & $\tilde{\chi}_{1}^{ \pm} q \bar{q}^{\prime}, \tilde{\chi}_{2}^{0} q \bar{q}, \tilde{\chi}_{1}^{0} q \overline{\bar{c}}$ \\
\hline$\tilde{d}_{L}$ & 1086.1 & $\tilde{\chi}_{1}^{-} u, \tilde{\chi}_{2}^{0} d$ & 1086.8 & $\tilde{\chi}_{1}^{-} u, \tilde{\chi}_{2}^{0} d$ & 1223.3 & 1224.2 & $\tilde{g} d, \tilde{\chi}_{1}^{-} u$ \\
\hline$\tilde{d}_{R}$ & 1043.4 & $\tilde{\chi}_{1}^{0} d$ & 1044.0 & $\tilde{\chi}_{1}^{0} d$ & 1217.3 & 1218.0 & $\tilde{g} d$ \\
\hline$\tilde{t}_{2}$ & 1035.7 & $\tilde{\chi}_{1}^{+} b, \tilde{\chi}_{4}^{0} t$ & 1008.4 & $\tilde{\chi}_{1}^{+} b, \tilde{\chi}_{4}^{0} t$ & 1031.5 & 961.8 & $\tilde{g} t, \tilde{\chi}_{1}^{+} b$ \\
\hline$\tilde{t}_{1}$ & 813.5 & $\tilde{\chi}_{1}^{+} b, \tilde{\chi}_{1}^{0} t, \tilde{\chi}_{2}^{+} b$ & 816.1 & $\tilde{\chi}_{1}^{+} b, \tilde{\chi}_{1}^{0} t, \tilde{\chi}_{2}^{+} b$ & 755.4 & 761.9 & $\tilde{\chi}_{2}^{+} b, \tilde{\chi}_{3}^{0} t$ \\
\hline$\underset{\sim}{b_{2}}$ & 1038.5 & $\tilde{\chi}_{2}^{-} t, \tilde{\chi}_{1}^{0} b$ & 1000.3 & $\tilde{\chi}_{2}^{-} t$ & 1204.5 & 1076.4 & $\tilde{g} b$ \\
\hline$b_{1}$ & 994.7 & $\tilde{\chi}_{1}^{-} t, \tilde{\chi}_{2}^{-} t, \tilde{\chi}_{2}^{0} b$ & 940.0 & $\tilde{\chi}_{1}^{-} t, \tilde{\chi}_{2}^{-} t, \tilde{\chi}_{2}^{0} b$ & 1016.5 & 941.1 & $\tilde{g} b, \tilde{\chi}_{2}^{-} t$ \\
\hline$\tilde{e}_{L}$ & 427.9 & $\tilde{\chi}_{1}^{0} e, \tilde{\chi}_{1}^{-} \nu$ & 427.9 & $\tilde{\chi}_{1}^{0} e, \tilde{\chi}_{1}^{-} \nu$ & 1111.7 & 1112.0 & $\tilde{\chi}_{1}^{-} \nu, \tilde{\chi}_{2}^{0} e$ \\
\hline$\tilde{e}_{R}$ & 321.2 & $\tilde{\chi}_{1}^{0} e$ & 321.6 & $\tilde{\chi}_{1}^{0} e$ & 1103.7 & 1103.7 & $\tilde{\chi}_{1}^{0} e$ \\
\hline$\tilde{\tau}_{2}$ & 426.9 & $\tilde{\chi}_{1}^{0} \tau, \tilde{\chi}_{1}^{-} \nu$ & 423.9 & $\tilde{\tau}_{1} Z / h, \tilde{\chi}_{1}^{0} \tau$ & 1107.5 & 1027.9 & $\tilde{\chi}_{1}^{-} \nu, \tilde{\chi}_{2}^{0} \tau$ \\
\hline$\tilde{\tau}_{1}$ & 316.3 & $\tilde{\chi}_{1}^{0} \tau$ & 213.6 & $\tilde{\chi}_{1}^{0} \tau$ & 1092.4 & 920.4 & $\tilde{\chi}_{1}^{0} \tau$ \\
\hline$\tilde{\chi}_{2}^{+}$ & 650.5 & $\tilde{\chi}_{2}^{0} W^{+}, \tilde{\chi}_{1}^{+} Z / h$ & 637.0 & $\tilde{\chi}_{2}^{0} W^{+}, \tilde{\chi}_{1}^{+} Z / h$ & 438.0 & 404.0 & $\tilde{\chi}_{2}^{0} W^{+}, \tilde{\chi}_{1}^{+} Z / h$ \\
\hline$\tilde{\chi}_{1}^{+}$ & 388.4 & $\tilde{\chi}_{1}^{0} W^{+}, \tilde{\tau} \nu$ & 390.6 & $\tilde{\tau}_{1} \nu$ & 200.4 & 201.1 & $\tilde{\chi}_{1}^{0} W^{+}$ \\
\hline$\tilde{\chi}_{4}^{0}$ & 651.0 & $\tilde{\chi}_{1}^{+} W^{-}, \tilde{\chi}_{2}^{0} h$ & 636.8 & $\tilde{\chi}_{1}^{+} W^{-}, \tilde{\chi}_{2}^{0} h$ & 438.1 & 403.4 & $\tilde{\chi}_{1}^{+} W^{-}, \tilde{\chi}_{2}^{0} h$ \\
\hline$\tilde{\chi}_{3}^{0}$ & 636.4 & $\tilde{\chi}_{1}^{+} W^{-}, \tilde{\chi}_{2}^{0} Z$ & 624.2 & $\tilde{\chi}_{1}^{+} W^{-}, \tilde{\chi}_{2}^{0} Z$ & 422.3 & 388.4 & $\tilde{\chi}_{1}^{+} W^{-}, \tilde{\chi}_{2}^{0} Z$ \\
\hline$\tilde{\chi}_{2}^{0}$ & 387.9 & $\tilde{\chi}_{1}^{0} h, \tilde{\tau}_{1} \tau$ & 390.0 & $\tilde{\tau}_{1} \tau$ & 199.8 & 200.4 & $\tilde{\chi}_{1}^{0} Z$ \\
\hline$\tilde{\chi}_{1}^{0}$ & 205.7 & stable & 206.3 & stable & 105.8 & 106.4 & stable \\
\hline
\end{tabular}


number of the background events for the search modes in Tab. 3. For the four jets with no lepton mode, the showed results are event numbers under the hardest cut in Tab. 3, which optimizes the significance for most of the model points.

Table 12: The number of signal events of the example model points for the five modes in Tab. 3 together with our estimation of the SM background. The event numbers are normalized for an integrated luminosity of $30 \mathrm{fb}^{-1}$ at the $7 \mathrm{TeV}$ run.

\begin{tabular}{|c|c|c|c|c|c|}
\hline & $0 \ell+2$ jets & $0 \ell+4$ jets & $2 \gamma$ & $2 \tau$ & SS $2 \ell$ \\
\hline \hline PT 1 & 0.3 & 0.5 & 45.3 & 0 & 0 \\
\hline PT 2 & 0 & 0.3 & 44.9 & 0.17 & 0 \\
\hline PT 3 & 9.1 & 6.0 & 0 & 6.04 & 63.5 \\
\hline PT 4 & 67.4 & 85.3 & 0 & 65.1 & 10.1 \\
\hline PT 5 & 0 & 10.7 & 0 & 10.7 & 57.8 \\
\hline PT 6 & 13.5 & 9.0 & 0 & 13.5 & 57.5 \\
\hline PT 7 & 310.4 & 79.0 & 1.1 & 1.14 & 10.3 \\
\hline PT 8 & 333.8 & 55.8 & 0 & 2.37 & 3.55 \\
\hline PT 9 & 71.1 & 94.0 & 0 & 2.30 & 34.4 \\
\hline PT 10 & 51.84 & 78.9 & 0 & 9.01 & 37.2 \\
\hline \hline SM BKG & 195.1 & 44.9 & 7.68 & 14.8 & 20.6 \\
\hline
\end{tabular}

\section{References}

[1] K. Kawagoe, T. Kobayashi, M. M. Nojiri and A. Ochi, Phys. Rev. D 69 (2004) 035003 hep-ph/0309031.

[2] M. Asano, T. Ito, S. Matsumoto and T. Moroi, arXiv:1111.3725 [hep-ph].

[3] See, for instance, M. Fairbairn, A. C. Kraan, D. A. Milstead, T. Sjostrand, P. Z. Skands and T. Sloan, Phys. Rept. 438, 1 (2007) arXiv:hep-ph/0611040], , and references therein.

[4] H. Pagels and J. R. Primack, Phys. Rev. Lett. 48 (1982) 223;

M. Viel, J. Lesgourgues, M. G. Haehnelt, S. Matarrese and A. Riotto, Phys. Rev. D

71 (2005) 063534 arXiv:astro-ph/0501562.

[5] E. Nakamura and S. Shirai, JHEP 1103 (2011) 115 [arXiv:1010.5995 [hep-ph]]. 
[6] K. Hamaguchi, S. Shirai and T. T. Yanagida, Phys. Lett. B 651, 44 (2007) arXiv:0705.0219 [hep-ph]], K. Hamaguchi, S. Shirai and T. T. Yanagida, Phys. Lett. B 663, 86 (2008) [arXiv:0712.2462 [hep-ph]].

[7] S. Frixione and B. R. Webber, JHEP 0206, 029 (2002) arXiv:hep-ph/0204244.

[8] M. L. Mangano, M. Moretti, F. Piccinini, R. Pittau and A. D. Polosa, JHEP 0307, 001 (2003) arXiv:hep-ph/0206293.

[9] J. Alwall et al., JHEP 0709, 028 (2007) [arXiv:0706.2334 [hep-ph]].

[10] T. Sjostrand, S. Mrenna and P. Z. Skands, JHEP 0605, 026 (2006) arXiv:hep-ph/0603175.

[11] E. Richter-Was, arXiv:hep-ph/0207355.

[12] J. T. Linnemann, Talk from PhyStat2003, Stanford, Ca, USA, September 2003 arXiv:physics/0312059

[13] G. Aad et al. [ATLAS Collaboration], arXiv:1109.6572 [hep-ex].

[14] A. Collaboration, arXiv:1109.6606 [hep-ex].

[15] CMS Collaboration, CMS Physics Analysis Summary CMS-PAS-SUS-11-010 (2011).

[16] CMS Collaboration, CMS Physics Analysis Summary CMSPAS-SUS-11-011 (2011).

[17] CMS Collaboration, CMS Physics Analysis Summary CMS-PAS-SUS-11-009 (2011).

[18] F. E. Paige, S. D. Protopopescu, H. Baer and X. Tata, arXiv:hep-ph/0312045].

[19] G. Marchesini, B. R. Webber, G. Abbiendi, I. G. Knowles, M. H. Seymour and L. Stanco, Comput. Phys. Commun. 67 (1992) 465;

G. Corcella et al., JHEP 0101 (2001) 010 arXiv:hep-ph/0011363;

G. Corcella et al., arXiv:hep-ph/0210213.

[20] G. Aad et al. [The ATLAS Collaboration], arXiv:0901.0512 [hep-ex].

[21] J. B. G. da Costa et al. [Atlas Collaboration], arXiv:1102.5290 [hep-ex].

[22] G. Aad et al. [Atlas Collaboration], arXiv:1102.2357 [hep-ex].

[23] G. Aad et al. [ATLAS Collaboration], arXiv:1103.6214 [hep-ex]. 
[24] N. Arkani-Hamed, G. L. Kane, J. Thaler, L. -T. Wang, JHEP 0608 (2006) 070. hep-ph/0512190. 